\title{
Semantical Mapping of Attribute Values for Data Integration
}

\author{
Marcin Szymczak*† Antoon Bronselaer ${ }^{\dagger}$ Sławomir Zadrożny* Guy De Tré ${ }^{\dagger}$ \\ * Systems Research Institute \\ Polish Academy of Sciences, Warsaw, Poland \\ $\dagger$ Department of Telecommunications and Information Processing \\ Ghent University, Ghent, Belgium
}

\begin{abstract}
Nowadays the amount of data is increasing very fast. Moreover, useful information is scattered over multiple sources. Therefore, automatic data integration that guarantees high data quality is extremely important. One of the crucial operations in integration of information from independent databases is detection of different representations of the same piece of information (called coreferent data) and translation of the representation of data from one source into the representation of the other source. That translation is also known as object mapping. In this paper, we investigate automatic mapping methods for attributes the values of which may need semantical comparison and can be sorted by means of an order relation that reflects a notion of generality. These mapping methods are investigated closely in terms of their effectiveness. An experimental evaluation of our method shows that using different mapping methods can enlarge a set of true positive mappings.
\end{abstract}

\section{INTRODUCTION}

\section{Problem statement}

One of the crucial issues to preserve high data quality in a database is the proper integration of data from different databases. Two major steps are considered in the data integration process. The first step is known as the schema matching problem which attempts at reconciling structural heterogeneity of data by mapping schema elements across the data sources [1], [2], [3], [4], [5], [6], [7]. The second step resolves semantic heterogeneity of data by mapping data instances across the datasets and is known as the object mapping problem [8], [9], [10], [11], [12], [13], [14], [15], [16].

In this paper, we present a novel approach for a specific part of the object mapping problem, namely we study automatic value mapping methods for attributes whose domains are partially ordered and where the given order relation reflects a notion of generality. We make the following three assumptions. First of all, the schema of each considered dataset contains an attribute which satifies the above condition, i.e. there exist a partial order relation defined on its domain. Next, such an order relation is known in advance. Finally, we assume that the schema matching between input datasets is established.

We consider two datasets as a running example in this contribution. They contain objects which are geographical annotations of a map that pinpoint locations of specific interest and are called points of interest (POIs). Each object is characterized by at least four attributes: name, longitude, latitude and category. The attribute name represents the identifier (name) of the specific POI, the longitude and latitude give the geographic coordinates of the place, and the category specifies the type or function of the location. The first dataset is represented by Table I which contains objects extracted from a Google database ${ }^{1}$, called the source $S$, with a known partial order relation on the domain of the category attribute. Figure 1 presents a part of this relation. The most general concept is a root of the tree and its descendant nodes correspond to narrower concepts. For instance, the concept establishment in Figure 1 is the most general concept for others values of attribute category of dataset $S$ and has children corresponding to more specific structures (e.g., lodging, etc.). The second dataset contains objects extracted from the RouteYou dataset $^{2}$, called the target $T$, also with a known partial order relation on the domain of the category attribute. Table II contains objects extracted from the target dataset, while a part of the order relation is presented in Figure 2. For instance, the concept $P O I$ in Figure 2 is the most general concept among the values of attribute category of dataset $T$ and has children corresponding to more specific concepts (e.g., Support, Accomodation, Shopping location, etc.).

TABLE I. EXAMPLE OF OBJECTS EXTRACTED FROM DATASET $S$.

\begin{tabular}{c|l|c|c|l}
\hline Id & Name & Lon. & Lat. & Category \\
\hline \hline 1 & Selfstorage-Achel & 5.470673 & 51.276789 & storage \\
2 & Campirama NV & 3.251893 & 50.852829 & campground \\
3 & Cafe-Restaurant De Ster & 4.050876 & 51.281777 & cafe \\
4 & Het Kouterhof & 3.665122 & 51.034331 & lodging \\
5 & Borluut Bed Breakfast & 3.657992 & 51.018882 & lodging \\
6 & Carlton Hotel & 3.713951 & 51.036280 & lodging \\
7 & Snooz Inn & 3.733049 & 51.058803 & lodging \\
\hline
\end{tabular}

TABLE II. EXAMPLE OF OBJECTS EXTRACTED FROM DATASET $T$.

\begin{tabular}{c|l|c|c|l}
\hline Id & Name & Lon. & Lat. & Category \\
\hline \hline 1 & Pakhuis Stokholm & 4.665898 & 51.818359 & Warehouse \\
2 & Camping De Iembarg & 7.111477 & 52.967909 & Camp Site \\
3 & Cafe Theatre & 3.722015 & 51.049830 & Restaurant \\
4 & Het Kouterhof & 3.665140 & 51.034379 & Hotel \\
5 & Borluut Bed Breakfast & 3.657975 & 51.018938 & Guest room \\
6 & Hotel City Inn & 9.369670 & 52.329782 & Hotel \\
7 & Santellone Resort & 10.16630 & 45.550103 & Hotel \\
\hline
\end{tabular}

Let us consider a data integration scenario in which objects from a dataset $S$ have to be merged with objects from a

\footnotetext{
${ }^{1}$ Google, http://maps.google.com

${ }^{2}$ RouteYou, http://www.routeyou.com/
} 


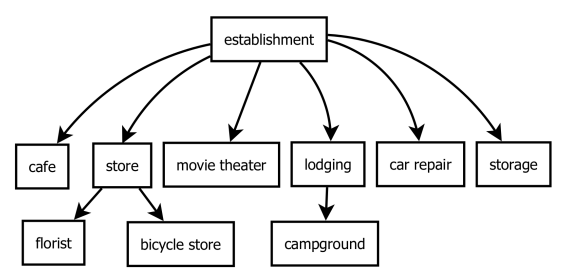

Fig. 1. A part of the partial order relation for the category attribute from the dataset $S$.

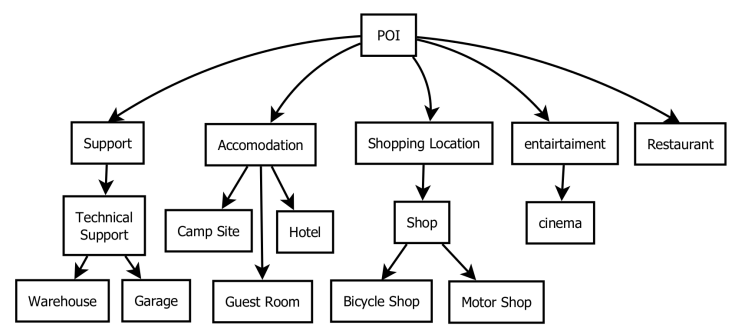

Fig. 2. A part of the partial order relation for the category attribute from the dataset $T$.

dataset $T$. Values of attribute as name, longitude and latitude might be stored in the target dataset $T$ without any additional processing assuming that there is no coreference between imported values and values of objects in the dataset $T$ for the corresponding attributes. However, importing of values of attributes such as category, representing information on the type of a point of interest, is less trivial as they may often refer to the same concepts presented in a different way in both datasets, called coreferent data. For instance, the concept accomodation is represented by the category lodging in the dataset $S$ and by the category Accomodation or Hotel in the dataset $T$. Therefore, for successful data integration, it may be crucial to create mappings of values of the category attribute in the datasets $S$ and $T$. Such mappings help to maintain consistency and decrease the number of duplicates in the integrated dataset which has extreme influence on data quality. This in turn descreases the cost of database maintenance.

In our approach, on the one hand, explicit mappings are created by predefined mappers which are based on category descriptions compared using information retrieval techniques. The category description is a textual description of each category and is generated from the values of other attributes (such as the name attribute) or extracted from an external source (i.e. World Wide Web). Moreover, the certainty of each mapping is expressed by a possibilistic truth value (PTV) and hence based on fuzzy set and possibility theory [17], [18]. On the other hand, the order relations and the explicit mappings are used to infer implicit mappings.

As a consequence there are established one-to-many mappings, which means that one category from the source dataset is mapped to one or more categories from the target dataset. The examples of mappings between categories forming hierarchies shown in Figure 1 and Figure 2 are presented in Figure 3. The dotted arrows indicate the mappings of categories from the source dataset to categories of the target dataset, e.g., the mapping of campground to Camp Site.

Many problems have to be addressed while devising such

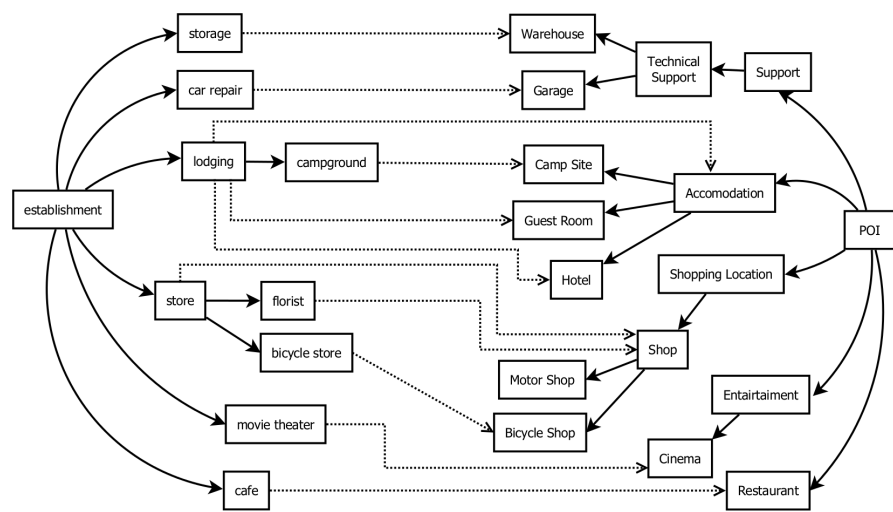

Fig. 3. Example of mappings between categories of dataset A and dataset B.

a mapping algorithm. The most important among them are the following:

- How to create a mapping between categories from heterogeneous sources?

- How can a partial order relation can be used to create a mapping between categories?

- Should the mapping be biased towards more specific or more general information?

\section{Paper outline}

The remainder of this paper is organized as follows. In Section II, overview of work related to the topic of this paper is provided. Next, in Section III some preliminary concepts are introduced that serve as a theoretical foundation of this paper. In Section IV a framework of value mapping is introduced and the algorithm is briefly described. Section V and Section VI contain the detailed description of applied mapping functions (mappers). Next, Section VII presents the results of computational experiments. Finally, we conclude and point out directions for a future work in Section VIII.

\section{RELATED WORK}

The problem of objects mapping has been studied in different contexts such as record linkage [12], duplicate detection [8], [19], [20], data integration [10] and knowledge base construction [21]. Most of the previous works assume that values of corresponding attributes are drawn from the same domain or at least that they bear some textual similarity that can be measured using a kind of the distance (e.g. edit distance, Jaccard). Moreover, some approaches are based on statistical information processing [11], [14], [16]. For instance, Kang et al. in [11] exploit a statistical model which captures the co-occurence of values of all attributes characterizing datasets. Next, constructed models are aligned assuming various matchings between the values of a given attribute in both datasets. The alignment with the minimum distance between thus aligned models is returned as the mapping. In [16] a strategy is presented which consists in the usage of statistical techniques to detect overlapping subsets of data present in disparate sources, through which rules for data conversion 
may be extracted. In [15] domain independent string transformations are proposed to compare syntactically object's shared attributes. The established mappings depend on the mapping rules which are determined by a mapping learner and supervised by the user. In contrast, in [9], mappings is based on non-overlapping correlated attributes using a combination of profilers which contain the specific knowledge about what constitutes a typical concept.

To the best of our knowledge, none of the mentioned approaches rely on a partial order relation to establish automatically semantical mappings for attributes values with different domains. In this paper, the order relation is used for the first time in the context of object mapping.

\section{PRELIMINARIES}

Within the scope of this paper it is assumed that entities from the real world are described as objects which are characterized by a number of attributes $a \in A$.

A schema of a given dataset is identified with the set of attributes $A$. For each attribute $a \in A$, let $\operatorname{dom}(a)$ denote the domain of $a$ (the set of possible values for attribute $a$ ).

\section{Problem Definition}

Two datasets are considered. The source dataset over the schema $A_{S}=\left\{a_{1}^{S}, \ldots, a_{n}^{S}\right\}$ is denoted as $S$, while the target dataset over the schema $A_{T}=\left\{a_{1}^{T}, \ldots, a_{m}^{T}\right\}$ is denoted as $T$. We assume that the schema matching is known:

$$
f: A_{S} \rightarrow A_{T}
$$

Moreover, at least one category attribute $a_{C}^{S} \in A_{S}$ is distinguished with values $c^{S} \in \operatorname{dom}\left(a_{C}^{S}\right)$ (called categories for short), and similarly $a_{C}^{T} \in A_{T}$ with values $c^{T} \in \operatorname{dom}\left(a_{C}^{T}\right)$. The category attribute of an object is an ordinal attribute [22] of which the values (categories) are (partially) ordered by means of a generalization/specialization relation. Besides that, the set of one-to-many categories mappings is defined as follows.

Definition 1 (One-To-Many Categories Mapping): The categories mapping is a one-to-many directional relation $R_{M 1: m}$ if it maps one category $c^{S} \in \operatorname{dom}\left(a_{C}^{S}\right)$ to a nonempty subset of categories $\left\{c_{1}^{T}, \ldots, c_{i}^{T}\right\} \subseteq \operatorname{dom}\left(a_{C}^{T}\right)$, called the candidate categories set, representing the coreferent categories of a pair of corresponding attributes $a_{C}^{S} \in A_{S}$ and $a_{C}^{T} \in A_{T}$, where $f\left(a_{C}^{S}\right)=a_{C}^{T}$ This mapping is denoted as:

$$
R_{M 1: m}: \operatorname{dom}\left(a_{C}^{S}\right) \longrightarrow_{1: m} \operatorname{range}\left(a_{C}^{T}\right)
$$

where $\operatorname{dom}\left(a_{C}^{S}\right)$ is a domain of the attribute $a_{C}^{S}$ and range $\left(a_{C}^{T}\right)$ is a subset of $\operatorname{dom}\left(a_{C}^{T}\right)$ comprising values of $a_{C}^{T}$. Thus, one-to-many categories mapping $R_{M 1: m}$ is a set of pairs $\left\{\left(c^{S}, c_{1}^{T}\right), \ldots,\left(c^{S}, c_{i}^{T}\right)\right\}$.

In our approach we use PTVs to express the confidence (certainty) in the validity of the mappings produced by an algorithm. Hereby, a PTV is a normalized possibility distribution [18] defined over the set of Boolean values $\mathbb{B}$ [17]. A PTV expresses the uncertainty about the Boolean value of a proposition $p$. In the context considered here, the propositions $p$ of interest are of the form:

$$
p=c^{S} \text { and } c^{T} \text { are coreferent }
$$

where $c^{S}$ and $c^{T}$ are two given categories, i.e., values of the category attributes from two datasets under consideration.

Let $P$ denote a set of all propositions under consideration. Then each $p \in P$ can be associated with a PTV denoted $\tilde{p}=\left\{\left(T, \mu_{\tilde{p}}(T)\right),\left(F, \mu_{\tilde{p}}(F)\right)\right\}$, where $\mu_{\tilde{p}}(T)$ represents the possibility that $p$ is true and $\mu_{\tilde{p}}(F)$ denotes the possibility that $p$ is false. The domain of all possibilistic truth values is denoted $\mathcal{F}(\mathbb{B})$, i.e., is the fuzzy power set of (normalised) fuzzy sets over $\mathbb{B}$.

Let us define the order relation $\geq$ on the set $\mathcal{F}(\mathbb{B})$ by:

$$
\tilde{p} \geq \tilde{q} \Longleftrightarrow\left\{\begin{array}{l}
\mu_{\tilde{p}}(F) \leq \mu_{\tilde{q}}(F), \quad \mu_{\tilde{p}}(T)=\mu_{\tilde{q}}(T)=1 \\
\mu_{\tilde{q}}(T) \leq \mu_{\tilde{p}}(T),
\end{array}\right.
$$

\section{MAPPING OF CATEGORIES}

Before we continue to describe our method to map values of category attributes, first of all we consider different types of mappings.

\section{A. Equivalent and Non-equivalent Mappings}

Let us consider our exemplary datasets $S$ and $T$ shown in Fig. 1 and Fig. 2, respectively. The mappings shown in Fig. 3 may be intuitively conceived. A group may be distinguished among these mappings which is represented by: movie theater can be mapped to Cinema, campground to Camp Site, car repair to Garage. These mappings are valid in both directions, i.e., from the dataset $S$ to the dataset $T$ and inversely, because these categories represent coreferent information on the same level of abstraction. These mappings are called equivalent mappings.

In contrast, mappings such as the one between concepts lodging and Hotel are asymmetric, in a sense. On the one hand, not each lodging is a Hotel. Therefore lodging should be mapped to a more general concept than Hotel. On the other hand, each Hotel is a lodging. These categories describe different levels of abstraction, they are not equivalent, i.e. lodging is more general concept than Hotel and Hotel is a specialization of lodging. Therefore, these mappings are called non-equivalent mappings which are further divided into two subclasses. The first one, called generalized mappings, contains mappings in which the target category is a generalization of the source category and it is a valid mapping but on a different level of abstraction. In contrast to that, a mapping of which the target category is a specialization of the source category is called specialized mapping and it may be an invalid mapping; however, between those categories there still exists a strong semantical relation.

Due to the above described conditions, the direction of mapping has to be considered during the data integration. In this paper we consider directional mappings from the source dataset $S$ to the target dataset $T$. 


\section{B. Algorithm}

Our method detects coreferent categories and establishes one-to-many relationship between them (in most cases) which is defined by Definition 1. An example of such a relationship (dotted arrows) is shown in Figure 3. Such a relationship can be further processed to reduce it to a one-to-one form. Such a processing as well as classification of a mapping as equivalent or non-equivalent may be needed in data integration, but this is out of the scope of this paper and it is studied, e.g., in [23].

The Category Mapping Algorithm (Algorithm 1) creates mappings for attributes equipped with a partial order relation that reflects a notion of generality. Therefore, a partial order relation $R_{S}$ on the domain of the source category attribute $a_{C}^{S} \in A_{S}$ and a partial order relation $R_{T}$ on the domain of the target category attribute $a_{C}^{T} \in A_{T}$ are assumed to be given. Moreover, given are also the datasets $S$ (source) and $T$ (target), and an extensible set of mapping methods, called mappers. These mappers are classified as explicit mappers $M_{E}$ and implicit mappers $M_{I}$ and are detailed in Section V and VI respectively. In the first step of our Algorithm 1 (lines 1-3) explicit mappings are established which are based on instance data of the datasets or external source. In the second step (lines 4-6) implicit mappings are inferred which are based on the explicit mappings and the partial order relations $R_{S}$ and $R_{T}$. The output of the algorithm is a relation $R_{M 1: m}$ stating coreference of categories.

The coreference of a pair of values is assumed to be a binary notion, i.e., two values are coreferent or not. However, one may be uncertain if it holds or not for a given pair of values. Thus, all the mappers associate each mapping they produce with uncertainty which is expressed by a PTV. Each mapping with PTV equal or close to $(1,0)$ is meant as holding with high confidence. In contrast, a mapping with PTV close to $(0,1)$ or $(1,1)$ means that the information about compared category values are not enough to claim relation between them. Therefore, only mappings associated with a PTV above predefined threshold for necessity of truth are taken into account.

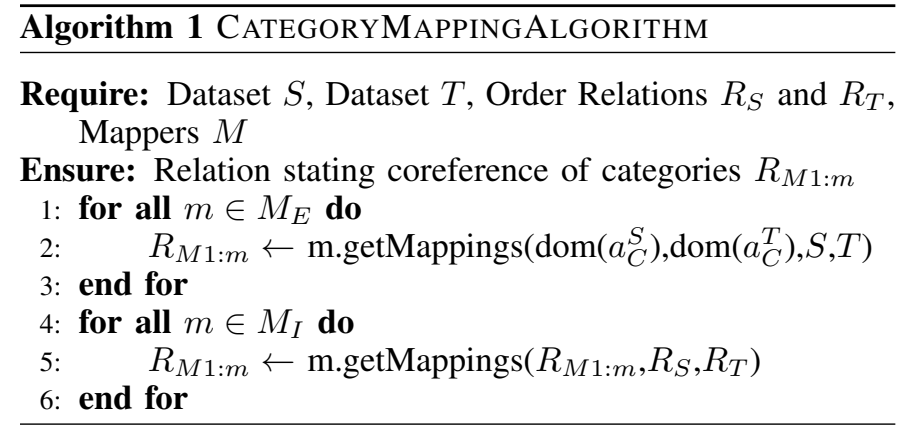

\section{EXPLICIT MAPPINGS}

The explicit mappings are created by description mappers and definition mappers which are based on different information. A description mapper is based on information about each category which is extracted from the source or target datasets. The definition mapper is based on information extracted from an external source (e.g., World Wide Web).
The considered mappers are based on a textual description of each category value (called category description) which is constructed from mapper-dependent information but in the similar way which is explained below.

\section{Category description}

The generation of the category description is divided into two phases: terms preprocessing and terms importance calculation. During the preprocessing phase, for each category value a representation in the form of a set of words/terms is obtained in the following way. The starting point is a collection of relevant strings, e.g., values of selected attributes, such as name in Tables I or II, of all objects belonging to the same category in the source/target dataset. First, special characters appearing in these strings, i.e. dash, semicolon, dot etc., are replaced by white space which gives a string of terms. Second, the strings are splitted into terms where the splitter is the white space character. Afterwards, each category is described by a set of terms which is further preprocessed by removing stop words and applying an algorithm for suffix stripping. Stop words are terms such as $a$, and or to in English. A popular predefined set of stop words contains 527 terms [24] and is used also in our computational experiments. Moreover, terms are stemmed using the Porter stemmer [25]. For instance, terms connection, connecting and connections are transformed to their stem which is connect.

The result of the term preprocessing phase is a clean and unified set of terms for each category value. The preprocessing increases the quality of terms importance calculation in the final phase of generating the category description. The importance of each term is expressed by the tfidf [26], [27] (term frequency and inversed document frequency) weighting scheme. This coefficient reflects that a term which occurs often but not in many other descriptions tend to be more relevant and informative than a term which appears in many descriptions. Tfidf weighs the frequency of a term $t$ in a description $d(t \in d)$ with a factor that discounts its importance with its appearances in the whole descriptions collection $D$ of the single dataset, which is defined as:

$$
t f i d f(t, d)=t f(t, d) \times \log \frac{|D|}{d f(t)}
$$

where $t f(t, d)$ is the frequency of term $t$ in the description $d$ and is expressed by Equation 5, $|D|$ is the size of the whole descriptions collection and $d f(t)$ is a number of descriptions in which term $t$ occurs (term $t$ occurs at least in one description).

$$
t f(t, d)=\frac{\operatorname{card}(t, d)}{\sum_{i}^{|d|} \operatorname{card}\left(t_{i}, d\right)}
$$

where $\operatorname{card}(t, d)$ is the cardinality of term $t$ in a description $d$ and is divided by the sum of cardinalities of all term from description $d$ to prevent a bias towards longer documents. Moreover, $t$ fidf weights are normalized as follow [26]:

$$
t f i d f^{*}(t, d)=\frac{t f i d f(t, d)}{\sqrt{\sum_{i}^{|d|} t f i d f_{i}^{2}}}
$$


where $t f i d f^{*}$ is the normalized $t f i d f$ and $t f i d f_{i}$ is the $t f i d f$ weight of a term from a description $d$. The final description category is represented as a set which consists of a unique term $t_{i}$ with a normalized weight $t f i d f_{i}^{*}$.

\section{A. Description Mapper}

The description mapper creates mappings based on the comparison of category descriptions of the source and the target categories which are generated using values of selected objects attributes. This mapper works as follows.

First of all, the category description for each category from the source and the target is constructed (see paragraph Category Description in Section V). To this aim, objects from the dataset are grouped into clusters of the same category. For each cluster, values of selected attributes are used to generate the category description (see paragraph Category Description in Section V). The selection of attributes can be done automatically by selecting all textual attributes. For some attributes, such as name, stemming is not employed because they contain many proper nouns which may mislead the algorithm.

Afterwards, constructed category descriptions of the source and target categories are pair-wise compared. This comparison estimates the possibility that two given categories (their descriptions) are coreferent and is based on intersection. More specifically, the detected common terms (with weights) of both descriptions are added to the intersection that is a common subset of both descriptions, while the remaining terms (with weights) are added to the subset called errors and have an influence on the computed possibility that categories are (not) coreferent.

This mapper generates a PTV which expresses the uncertainty about the coreference of the compared descriptions as described above. The possibility that a proposition $p$, stating that two categories are coreferent, is true $\left(\mu_{\tilde{p}}(T)\right)$ and the possibility that $p$ is false $\left(\mu_{\tilde{p}}(F)\right)$ are calculated by the following equations:

$$
\begin{aligned}
& \mu_{\tilde{p}}(T)=\frac{\text { poss } T}{\text { factor }} \\
& \mu_{\tilde{p}}(F)=\frac{\text { poss } F}{\text { factor }}
\end{aligned}
$$

where possT, poss $F$ and factor are equal:

$$
\begin{gathered}
\operatorname{poss} T=\left(\sum_{i=1}^{\mid \text {intersection } \mid} \frac{\operatorname{tfidf}_{i}^{* S}+t f i d f_{i}^{* T}}{2}\right)^{\text {pow }} \\
\text { poss } F=\sum_{i=1}^{\mid \text {errors } \mid} t f i d f_{i}^{*} \\
\text { factor }=\max (\text { poss } T, \text { poss } F)
\end{gathered}
$$

where $\mid$ intersection $\mid$ denotes the number of common terms, and $\mid$ errors $\mid$ is the number of the remaining terms present in the representation of the source and target categories, $t f i d f_{i}^{* S}$ $\left(t f i d f_{i}^{* T}\right)$ is a weight of the common term from the description of the category from the source (the target respectively) and $t f i d f_{i}^{*}$ is a weight of a remaining term from the description of the category from the source or the target.

On the one hand, possT is the sum of the average of common terms weights and raised to the predefine power pow which is set for this mapper to 3. On the other hand, poss $F$ is computed as the sum of the weights $t f i d f_{i}^{*}$ of the remaining terms (from errors that are found during comparison). Finally, factor is the maximum of poss $T$ and poss $F$ and is used to normalize both possibilities. If the PTV of a mapping of any category description of the source and any category description of the target is above the predefined threshold then the mapping between considered categories is added to $R_{M 1: m}$.

For instance, consider categories lodging from the source and Hotel from the target. Let the source dataset contain objects with category lodging as shown in Table I. A part of the description of lodging contains the following terms with tfidf*: Hotel 0.85, Bed 0.22, Breakfast 0.22, Resort 0.08, Inn 0.03, Carlton 0.012, Borluut 0.006, etc. On the one hand, terms Carlton and Borluut get low weights because they appear infrequently in objects of category lodging and, moreover, they are not specific for this category. On the other hand, terms Bed and Hotel get higher weights because they are specific terms for accomodation and they appear in many objects of this category. Meanwhile, a part of description of the target category Hotel which is based on the names of objects from the target, shown in Table II, is the following: Hotel 0.96, Inn 0.14, Resort 0.08, Bed 0.01, Breakfast 0.01, Carlton 0.005, etc. Afterwards, the sets are compared and the common terms are detected. Finally, the PTV of this mapping is calculated using Equations (7) and (8) and equals $(1,0.06)$ which supports high confidence in the coreference relationship between the considered categories.

\section{B. Definition Mapper}

In contrast to the previous mapper, this method is based on the extraction of additional knowledge from an external source. More precisily, for each category the textual description is extracted from the Web, in particular, from the Wikipedia ${ }^{3}$. The textual description is a webpage (e.g., for the category lodging it is a webpage at the URL http://en.wikipedia.org/wiki/lodging) which is parsed by JSoup HTML Parser ${ }^{4}$. Extracted texts are used to construct the category description (see paragraph Category Description in Section V). Afterwards, the category descriptions are pair-wise compared and uncertainty as to their matching is quantified like in case of the description mapper but with the parameter pow in (9) set to 4 what have been experimentally confirmed to make the results of both types of the mappers comparable.

The extraction works as follows. First of all, for each category a predefined number of paragraphs from a relevant Wikipedia web page is extracted. Next, hyperlinks present in the extracted paragraphs are followed and further paragraphs are extracted from the target web pages. This is continued until the predefined reference level is reached. The reference

\footnotetext{
${ }^{3}$ Wikipedia, http://www.en.wikipedia.org

${ }^{4}$ JSoup HTML Parser, http://www.jsoup.org
} 
level states the limit of such a recursive hyperlinks following. We set the reference level to 1 and the number of extracted paragraphs to 2 based on experimental results.

For instance, a part of the extracted textual description of category lodging from the source dataset is the following (terms in bold refer to linked pages and for them recursive extraction was executed):

Lodging (or a holiday accommodation) is a type of residential accommodation (author's note: refer to dwelling). People who travel and stay away from home for more than a day need lodging for sleep, rest, safety, shelter from cold temperatures or rain, storage of luggage and access to common household functions. Lodging is done in a hotel, motel, hostel or hostal, a private home (commercial, i.e. a bed and breakfast, a guest house, a vacation rental, or non-commercially, with members of hospitality services or in the home of friends), in a tent, caravan/camper (often on a campsite) (... $)^{5}$.

While a part of the textual description of category Accomodation from the target is the following:

Accomodation may refer to: a dwelling, a place of temporary lodging (... $)^{6}$. A dwelling (also residence, abode) is an important legal concept which defines a self-contained unit of accommodation used by one or more households as a home, such as a house, apartment, (... $)^{7}$.

These texts are used to generate category descriptions. A part of the description of lodging contains the following most important terms (their stems) with weights: lodg 0.3957, backpack 0.3359, hous 0.3051, room 0.2277, accommod 0.2257, residenti 0.099 , facil 0.078 , home 0.081 , household 0.064 , etc. While a part of the description of the category Accommodation from the target is the following: home 0.3977, lodg 0.3808 , accommod 0.1458 , household 0.1434 , residenti 0.131 , facil 0.041 , etc. The descriptions comparison of lodging and Accommodation returns the mapping with PTV equal $(1,0.17)$. The uncertainty about this mapping is low because the description of Accomodation contains the description of lodging. Thus, the results confirm intuition that lodging is coreferent to accomodation.

\section{IMPLICIT MAPPINGS}

Besides the explicit mappings, our method returns implicit mappings (lines 4-6 in Algorithm 1). We consider two types of implicit mappings. The first type of implicit mapping (Implicit Mapping I) is established when for the specific category $c_{1}^{S}$ from the source there does not exist any explicit mapping. It is a mapping of the first one-level more general concept $c_{2}^{S}$ in a partial order relation $R_{S}$ of the considered category $c_{1}^{S} \in \operatorname{dom}\left(a_{C}^{S}\right)$ from the source dataset for which there exists an explicit mapping $m \in R_{M 1: m}$. The implicit mapping for $c_{1}^{S}$ is thus $c^{T} \in \operatorname{dom}\left(a_{C}^{T}\right)$ such that $c^{T}$ is the target category to which $c_{2}^{S}$ is mapped via $m$. It should be stressed that the quality of the implicit mappings depends on the correctness of explicit mappings and the order relation.

\footnotetext{
${ }^{5}$ The text is extracted from the definition of lodging in Wikipedia, http://en.wikipedia.org/wiki/Lodging

${ }^{6}$ The text is extracted from the definition of Accomodation in Wikipedia, http://en.wikipedia.org/wiki/Accomodation

${ }^{7}$ The text is extracted from the definition of $d$ welling in Wikipedia, http://en.wikipedia.org/wiki/Dwelling
}

For instance, $R_{S}$ in Figure 1 contains the following pairs of elements: (establishment, store), (store, florist), where establishment is the most general concept. Suppose that the set of already detected mappings contains mapping (store, Shop). If the category florist is not mapped explicitly then the algorithm returns an implicit mapping of florist to Shop.

Moreover, the set of mappings is extended by the second type of implicit mappings (Implicit Mapping II) which work as follow. For each target category of a mapping categories from the target partial order relation $R_{T}$ which are generalizations of the considered category are extracted. Next, there are established mappings between the considered category and extracted generalizations.

For instance, suppose that there exists mapping between lodging from the source and Hotel from the target. Using the order relation shown in Fig. 2 the generalizations of Hotel are categories Accomodation and POI. Thus, mappings are established between them and the source category lodging.

\section{EVALUATION AND DISCUSSION}

The evaluation of our algorithm is conducted based on two real-world datasets. The source dataset contains around 200000 objects and a partial order relation on the set of 100 categories which are extracted from the Google Maps database by the Google Places API ${ }^{6}$. The target contains around 430000 objects and a partial order relation on the set of 502 categories which are shared by RouteYou ${ }^{7}$.

Our algorithm employs two thresholds to decide on categories coreference which are set to 0.2 for $\mu_{\tilde{p}}(F)$ of Definition Mapper (0.1 of Description Mapper respectively) and to 0.5 for $\mu_{\tilde{p}}(T)$ of both mappers based on experimental results. If $\mu_{\tilde{p}}(F)$ is lower than the threshold then the coreference is declared. If $\mu_{\tilde{p}}(T)$ is lower than the threshold then the lack of coreference is declared. Finally, if both of the thresholds are exceeded then the coreference status is declared as unknown.

For both datasets in total our algorithm suggested 263 mappings between the categories from the source and the target using mappers which are described in Sections V and VI. A part of the results is presented in Table III. Each mapping consists of four values: the source category, the target category, PTV and the name of the mapper which produced the specific mapping. There can be distinguished equivalent mappings (i.e. lodging and Accomodation, establishment and $P O I$ ), generalized non-equivalent mappings (i.e. church and Place of Worship) and specialized non-equivalent mappings (i.e. lodging and Hotel).

The quality of our method is evaluated using two standard measures of recall and precision. The precision is a fraction of detected real coreferent mappings among all detected mappings, the recall is a number of detected real coreferent mappings divided by the number of all real coreferent mappings. The sets of possible real coreferent equivalent and non-equivalent mappings are provided manually by experts. Table IV presents the quality measures of our approach for equivalent mappings (column 2), equivalent and generalized

\footnotetext{
${ }^{6}$ Google Places, http://developers.google.com/places/

${ }^{7}$ Route You, http://routeyou.com/
} 
non-equivalent mappings (column 3), equivalent and all nonequivalent mappings (column 4).

Additionally, the last column in Table IV contains of recall and precision for any but not the most general mappings. They show if there is established at least one coreferent mapping for each category from the source which is not a mapping for the most general category in the order relation $R_{S}$, called root which is $P O I$ for $R_{S}$ (by Definition 1 each category from the source can be mapped to the root). Thus, in this case the precision and recall are calculated as follows. The precision is a number of distinct source categories (different from the root) of detected real coreferent mappings divided by the number of distinct source categories (different from the root) of all detected mappings, the recall is a number of distinct source categories (different from the root) of detected real coreferent mappings divided by the number of distinct source categories (different from the root) of all real coreferent mappings.

Firstly, the recall and the precision in Table IV are calculated for explicit mappers (above the bar), for which high thresholds are set. As consequence they return high precision but low recall for equivalent and non-equvalent mappings (column 4). The high precision is important because these mappings are used by the implicit mappers: any false positive mappings are propagated by implicit mappers what lowers the overall result. Besides that, it is not crucial to detect all posible coreferent mappings but it is sufficient if the algorithm creates at least one coreferent mapping for each category which turns out to be the proper mapping (the selection of mappings is out of the scope of this paper and is investigated in [23]). The results in the last column confirm that, for around half of the categories from the source at least one coreferent mapping is established which is different from root with the precision equals 0.93 .

Next, the recall and the precision are calculated for explicit and implicit mappings combined. The implicit mappers decrease the precision for equivalent mappings (column 1 in Table IV) because they create mostly the non-equivalent mappings. Thus, for non-equivalent mappings (column 3 and 4 in Table IV) these mappers increase the precision and the recall, i.e. the precision from 0.41 to 0.57 and the recall from 0.11 to 0.4 for equivalent and generalized mappings (column 3 ); the precision from 0.78 to 0.79 and the recall from 0.12 to 0.33 for equivalent and non-equivalent mappings (column 4 ). Besides that, for almost half of categories from the source there is established at least one coreferent mapping which is different from root with the precision equals 0.98 .

\section{CONCLUSION AND FUTURE WORK}

We present a novel automatic method to establish oneto-many semantical mappings between attributes values from different domains. This method applies an extensible set of mappers which are based on the constructed textual descriptions of considered values and employs information retrieval techniques for further processing. Moreover, we have also shown how a known partial order relation defined on the domain of considered attributes can be used to create mappings.

Our approach generates the set of mappings where each value from the source dataset is related to at least one value from the target dataset. It, in general, produces alternative
TABLE III. SOME RESULTS OF ALGORITHM: MAPPINGS OF VALUES FROM THE SOURCE AND TARGET DATASETS.

\begin{tabular}{|c|c|c|c|}
\hline Category from $S$ & Category from $T$ & PTV & Mapper \\
\hline art gallery & Art Museum & $(1,0)$ & Definition \\
\hline art gallery & Museum & $(1,0)$ & ImplicitII \\
\hline art gallery & Cultural Centre & $(1,0)$ & ImplicitII \\
\hline art gallery & Tourist Attract & $(1,0)$ & ImplicitII \\
\hline art gallery & Recreation & $(1,0)$ & ImplicitII \\
\hline art gallery & Building Constr & $(1,0)$ & ImplicitII \\
\hline art gallery & POI & $(1,0)$ & ImplicitII \\
\hline restaurant & Eatery & $(1,0)$ & Definition \\
\hline restaurant & Eat and Drink & $(1,0)$ & ImplicitII \\
\hline restaurant & POI & $(1,0)$ & ImplicitII \\
\hline restaurant & Road Restaurant & $(1,0.003)$ & Definition \\
\hline restaurant & Hotel & $(1,0.09)$ & Description \\
\hline amusement park & Themepark & $(1,0)$ & Definition \\
\hline amusement park & Amusement Park & $(1,0)$ & Definition \\
\hline amusement park & Recreation & $(1,0)$ & ImplicitII \\
\hline amusement park & POI & $(1,0)$ & ImplicitII \\
\hline church & church & $(1,0)$ & Definition \\
\hline church & Place of Worship & $(1,0)$ & ImplicitII \\
\hline church & POI & $(1,0)$ & ImplicitII \\
\hline church & Place of Worship & $(1,0.1)$ & Description \\
\hline storage & Warehouse & $(1,0.001)$ & Definition \\
\hline storage & POI & $(1,0.001)$ & ImplicitII \\
\hline establishment & POI & $(1,0.015)$ & Description \\
\hline natural feature & Waterfall & $(1,0.05)$ & Definition \\
\hline natural feature & Landscape Element & $(1,0.05)$ & ImplicitII \\
\hline natural feature & POI & $(1,0.05)$ & ImplicitII \\
\hline natural feature & Dunes & $(1,0.12)$ & Definition \\
\hline lodging & Hotel & $(1,0.06)$ & Description \\
\hline lodging & Accomodation & $(1,0.06)$ & ImplicitII \\
\hline lodging & Accom Shelter & $(1,0.06)$ & ImplicitII \\
\hline lodging & POI & $(1,0.06)$ & ImplicitII \\
\hline lodging & Accomodation & $(1,0.17)$ & Definition \\
\hline food & Restaurant & $(1,0.1)$ & Description \\
\hline food & Eatery & $(1,0.1)$ & ImplicitII \\
\hline food & Eat and Drink & $(1,0.1)$ & ImplicitII \\
\hline food & POI & $(1,0.1)$ & ImplicitII \\
\hline meal delivery & Restaurant & $(1,0.1)$ & Implicit \\
\hline meal delivery & Eatery & $(1,0.1)$ & ImplicitII \\
\hline meal delivery & Horeca & $(1,0.1)$ & ImplicitII \\
\hline meal delivery & Eat and Drink & $(1,0.1)$ & ImplicitII \\
\hline meal delivery & POI & $(1,0.1)$ & ImplicitII \\
\hline
\end{tabular}

mappings for the same value. However, it may be crucial to select for each value from the source exactly one from the target. Thus, more sophisticated selection method than the method which is based on the lowest uncertainty about mapping have to be investigated [23]. Moreover, our method can be easily applied for data integration or interoperability tasks.

In the future we plan to extend the set of mappers, e.g., by a duplicates detection based mapper or a domain specific mapper which will be able to exploit also other attributes such as those representing the coordinates of an object. This should increase the number of detected correct mappings and improve the quality of our approach.

\section{ACKNOWLEDGMENT}

This contribution is supported by the Foundation for Polish Science under International $\mathrm{PhD}$ Projects in Intelligent Com- 
TABLE IV. Results of the EVAluation: PRecision and Recall.

\begin{tabular}{|c|c|c|c|c|c|c|c|c|}
\hline \multirow[t]{2}{*}{ Mapper } & \multicolumn{2}{|c|}{ Equivalent } & \multicolumn{2}{|c|}{ Equivalent \& Generalized } & \multicolumn{2}{|c|}{ Equivalent \& Non-equivalent } & \multicolumn{2}{|c|}{ Any Not The Most General } \\
\hline & Precision & Recall & Precision & Recall & Precision & Recall & Precision & Recall \\
\hline Description & 0.13 & 0.03 & 0.31 & 0.01 & 0.94 & 0.02 & 1.00 & 0.09 \\
\hline Definition & 0.39 & 0.52 & 0.44 & 0.10 & 0.76 & 0.10 & 0.92 & 0.39 \\
\hline Desc., Def. & 0.34 & 0.54 & 0.41 & 0.11 & 0.78 & 0.12 & 0.93 & 0.45 \\
\hline Desc., Implicit I & 0.09 & 0.03 & 0.32 & 0.02 & 0.77 & 0.03 & 0.83 & 0.11 \\
\hline Desc., Implicit II & 0.09 & 0.08 & 0.40 & 0.06 & 0.93 & 0.08 & 1.00 & 0.09 \\
\hline Desc., Implicit I \& II & 0.06 & 0.08 & 0.41 & 0.09 & 0.78 & 0.10 & 0.83 & 0.11 \\
\hline Def., Implicit I & 0.39 & 0.52 & 0.44 & 0.10 & 0.76 & 0.10 & 0.92 & 0.39 \\
\hline Def., Implicit II & 0.17 & 0.56 & 0.63 & 0.35 & 0.77 & 0.25 & 0.97 & 0.41 \\
\hline Def., Implicit I \& II & 0.19 & 0.56 & 0.63 & 0.35 & 0.77 & 0.25 & 0.97 & 0.41 \\
\hline Desc., Def., Implicit I & 0.33 & 0.54 & 0.42 & 0.12 & 0.78 & 0.13 & 0.93 & 0.47 \\
\hline Desc., Def., Implicit II & 0.15 & 0.59 & 0.58 & 0.38 & 0.79 & 0.32 & 0.98 & 0.47 \\
\hline All & 0.14 & 0.59 & 0.57 & 0.40 & 0.79 & 0.33 & 0.98 & 0.49 \\
\hline
\end{tabular}

puting. Project financed from The European Union within the Innovative Economy Operational Programme 2007-2013 and European Regional Development Fund.

\section{REFERENCES}

[1] E. Rahm and P. A. Bernstein, "A survey of approaches to automatic schema matching," The VLDB Journal, vol. 10, no. 4, pp. 334-350, Dec. 2001.

[2] M. Szymczak, S. Zadrozny, and G. De Tré, "Coreference detection in xml metadata," in 2013 Joint IFSA World Congress NAFIPS Annual Meeting, Proceedings, W. Pedrycz and M. Reformat, Eds., 2013, pp. 1354-1359.

[3] J. Madhavan, P. A. Bernstein, and E. Rahm, "Generic schema matching with cupid," in Proceedings of the 27th International Conference on Very Large Data Bases, ser. VLDB '01. San Francisco, CA, USA: Morgan Kaufmann Publishers Inc., 2001, pp. 49-58.

[4] H. hai Do and E. Rahm, "Coma - a system for flexible combination of schema matching approaches," in In VLDB, 2002, pp. 610-621.

[5] A. Bilke and F. Naumann, "Schema matching using duplicates," in Proceedings of the 21st International Conference on Data Engineering, ICDE 2005, 5-8 April 2005, Tokyo, Japan. IEEE Computer Society, 2005, pp. 69-80.

[6] R. Dhamankar, Y. Lee, A. Doan, A. Halevy, and P. Domingos, "imap: discovering complex semantic matches between database schemas," in in: Proceedings of the 2004 ACM SIGMOD International Conference on Management of Data, ACM. Press, 2004.

[7] M. Szymczak and J. Koepke, "Matching methods for semantic annotation-based xml document transformations," in K. Atanassov, et al. (Eds.), New Developments in Fuzzy Sets, Intuitionistic Fuzzy Sets, Generalized Nets and Related Topics. Applications. Volume II. SRI PAS, 2012, pp. 297-308.

[8] R. Ananthakrishna, S. Chaudhuri, and V. Ganti, "Eliminating fuzzy duplicates in data warehouses," in Proceedings of the 28th International Conference on Very Large Databases (VLDB 2002), 2002.

[9] A. Doan, Y. Lu, Y. Lee, and J. Han, "Object matching for information integration: A profiler-based approach," in In: Proceedings of the IJCAI03 Workshop on Information Integration on the Web. (2003, 2003, pp. 53-58.

[10] F. Naumann, A. Bilke, J. Bleiholder, and M. Weis, "Data fusion in three steps: Resolving inconsistencies at schema-, tuple-, and valuelevel," in IN BULLETIN OF THE TECHNICAL COMMITTEE ON DATA ENGINEERING, 2006, pp. 21-31.

[11] J. Kang, D. Lee, and P. Mitra, "Identifying value mappings for data integration: An unsupervised approach." in WISE, ser. Lecture Notes in Computer Science, A. H. H. Ngu, M. Kitsuregawa, E. J. Neuhold, J.-Y. Chung, and Q. Z. Sheng, Eds., vol. 3806. Springer, 2005, pp. 544-551.

[12] I. P. Fellegi and A. B. Sunter, "A theory for record linkage," Journal of the American Statistical Association, vol. 64, pp. 1183-1210, 1969.
[13] M. Craven, D. Dipasquo, D. Freitag, A. Mccallum, T. Mitchell, and K. Nigam, "Learning to construct knowledge bases from the world wide web," Artificial Intelligence, vol. 118, pp. 69-113, 1999.

[14] W. W. Cohen, "Integration of heterogeneous databases without common domains using queries based on textual similarity," 1998, pp. 201-212.

[15] S. Tejada, C. A. Knoblock, and S. Minton, "Learning domainindependent string transformation weights for high accuracy object identification," in Proceedings of the eighth ACM SIGKDD international conference on Knowledge discovery and data mining, ser. KDD '02. New York, NY, USA: ACM, 2002, pp. 350-359.

[16] H. Lu, W. Fan, C. H. Goh, S. E. Madnick, and D. W.-L. Cheung, "Discovering and reconciling semantic conflicts: A data mining perspective." in DS-7, 1997, pp. 409-427.

[17] H. Prade, "Possibility sets, fuzzy sets and their relation to Lukasiewicz logic," in Proc 12th Int Symp on Multiple-Valued Logic, 1982, pp. 223227.

[18] L. A. Zadeh, "Fuzzy sets as a basis for a theory of possibility," Fuzzy Sets Syst., vol. 100, pp. 9-34, Apr. 1999.

[19] M. Weis and F. Naumann, "Detecting duplicate objects in xml documents." in IQIS, F. Naumann and M. Scannapieco, Eds. ACM, 2004, pp. $10-19$.

[20] _ "Dogmatix tracks down duplicates in xml." in SIGMOD Conference, F. zcan, Ed. ACM, 2005, pp. 431-442.

[21] M. Craven, D. DiPasquo, D. Freitag, A. McCallum, T. Mitchell, K. Nigam, and S. Slattery, "Learning to construct knowledge bases from the world wide web," Artificial Intelligence, vol. 118, pp. $69-$ 113, 2000.

[22] S. S. Stevens, "On the theory of scales of measurement," Science, vol. 103, no. 2684, pp. 677-680, 1946.

[23] M. Szymczak, A. Bronselaer, S. Zadrozny, and G. De Tré, "Selection of semantical mappings of attribute values for data integration," in To appear in IEEE Intelligent Systems 2014, Proceedings. Springer, 2014, pp. $1-12$.

[24] M. Hall, E. Frank, G. Holmes, B. Pfahringer, P. Reutemann, and I. H. Witten, "The weka data mining software: An update," SIGKDD Explor. Newsl., vol. 11, no. 1, pp. 10-18, Nov. 2009.

[25] M. F. Porter, "Readings in information retrieval," K. Sparck Jones and P. Willett, Eds. San Francisco, CA, USA: Morgan Kaufmann Publishers Inc., 1997, ch. An Algorithm for Suffix Stripping, pp. 313-316.

[26] G. Salton and C. Buckley, "Term weighting approaches in automatic text retrieval," Ithaca, NY, USA, Tech. Rep., 1987.

[27] W. B. Frakes and R. Baeza-Yates, Eds., Information Retrieval: Data Structures and Algorithms. Upper Saddle River, NJ, USA: PrenticeHall, Inc., 1992. 